\title{
GAMES AND SUB-GAMES
}

\section{SEYMOUR SHERMAN}

The determination of the value and optimal strategies of a zerosum, two-person game with a finite number of pure strategies can be a lengthy process, involving, among other things, the calculation of the value

$$
\sup _{x} \inf (x B, y)
$$

where $B$ is a real matrix with $m$ rows and $n$ columns, $x$ ranges over the set of row vectors with $m$ components, all non-negative and adding up to one, $y$ ranges over the corresponding set of $n$-component column vectors, and the pay-off, $(x B, y)$, indicates the inner product of the two vectors $x B$ and $y$. One device which may simplify a game computation is that of "dominance" or "majorization" [vNM, p. 174] by which the solution of a game is reduced to the solution of a smaller game, that is, one with a smaller number of pure strategies. There is another device which, when conditions are right, may simplify the solution of a game by reducing it to the solution of smaller games. This device, presented here, gives either the value or a bound for it, depending on the information available about the sub-games. It also gives an optimal strategy or a strategy sufficient to insure an outcome not worse than that predicted by the aforementioned bound. It is particularly effective when there are rows (or columns) in $B$, which are constant or have large constant segments.

Let $B$ be a game matrix (rows maximizing) decomposed into

$$
B=\left\{B_{j}^{i} \mid 1 \leqq i \leqq M, 1 \leqq j \leqq N\right\},
$$

where $B_{j}^{i}$ is a sub-matrix with $m_{i}$ rows and $n_{j}$ columns (the $m_{i}$ rows being independent of $j$ and the $n_{j}$ columns being independent of $i$ ). Let the value of $B$ be $v$ and the value of $B_{j}^{i}$ be $v_{j}^{i}$. Let the set of optimal strategies for the first player in the game be $X=\{x\}$ and the set of optimal strategies for the first player in the sub-game be $X_{j}^{i}=\left\{x^{i}\right\}$. Let $Y$ and $Y_{j}^{i}$ represent the corresponding sets for the

Presented to the Society, April 29, 1950; received by the editors February 22, 1950.

1 This originally appeared in a RAND report: Total reconnaissance with total countermeasures: Simplified model, August 5, 1949, P-106, Rand Corporation, Santa Monica, California. For the definitions in game theory see [vNM]. See the bibliography at the end of the paper. 
second players. Let $\bar{B}$ be the $M \times N$ matrix with entries $v_{j}^{i}$. Let $\bar{x}=\left\{\bar{x}_{i} \mid 1 \leqq i \leqq M, \bar{x}_{i} \geqq 0, \sum \bar{x}_{i}=1\right\}$ be a typical optimal strategy for the first player in the game with matrix $\bar{B}, \bar{X}$ the set of optimal strategies for the first player in $\bar{B}$, and $\bar{y}$ and $\bar{Y}$ the analogous items for the second player. Let $\bar{v}$ be the value of the game with matrix $\bar{B}$.

TheOREm. $\bigcap_{j} X_{j}^{i} \neq \Lambda$ for each $i$ implies $v \geqq \bar{v}$. If $x^{i} \in \bigcap_{j} X_{j}^{i}$ for each $i$ and $\bar{x} \in \bar{X}$, then by playing the vector $\left\{\bar{x}_{1} x^{1}, \bar{x}_{2} x^{2}, \cdots, \bar{x}_{M} x^{M}\right\}$ (where by this notation we mean the vector each of whose first $m_{1}$ components are $\bar{x}_{1}$ multiplied by the appropriate one of the $m_{1}$ components of $x^{1}$, and so on) the first player may assure himself of a pay-off of at least $\bar{v}$.

Proof. Let a typical strategy for II in game with matrix $B$ be

$$
y=\left\{\beta^{1} \tilde{y}_{1}, \cdots, \beta^{N} \tilde{y}_{N}\right\}
$$

where $\tilde{y}_{j}$ is a vector with $n_{j}$ non-negative components adding up to one and $\beta^{j} \geqq 0$ for each $j, \sum \beta^{j}=1$. If I plays

$$
\left\{\bar{x}_{1} x^{1}, \cdots, \bar{x}_{M} x^{M}\right\}
$$

then the pay-off

$$
\sum_{j=1}^{N}\left(\sum_{i=1}^{M} \bar{x}_{i} x^{i} B_{j}^{i}, \beta^{j} \tilde{y}_{j}\right) \geqq \sum_{j=1}^{N}\left(\sum_{i=1}^{M} \bar{x}_{i} v_{j}^{i} \beta^{j}\right) \geqq \bar{v} .
$$

Corollary. $\bigcap_{i} Y_{j}^{i} \neq \Lambda$ for each $j$ implies $v \leqq \bar{v}$. If $y_{j} \in \bigcap_{i} Y_{j}^{i}$ for each $j$ and $\bar{y} \in \bar{Y}$, then by playing the vector $\left\{\bar{y}^{1} y_{1}, \cdots, \bar{y}^{N} y_{N}\right\}$ the second player may limit his losses to $\bar{v}$.

Corollary. ${ }^{2} \bigcap_{j} X_{j}^{i} \neq \Lambda$ for each $i$ and $\bigcap_{i} Y_{j}^{i} \neq \Lambda$ for each $j$ implies $v$ $=\bar{v}$. $x^{i} \in \bigcap_{j} X_{j}^{i}, y_{j} \in \bigcap_{i} Y_{j}^{i}, \bar{x} \in \bar{X}$ and $\bar{y} \in \bar{Y}$ implies $\left\{x_{1} x^{1}, \cdots, x_{M} x^{M}\right\}$ $\in X$ and $\left\{\bar{y}^{1} y_{1}, \cdots, \bar{y}^{N} y_{N}\right\} \in Y$.

\section{BIBLIOGRAPHY}

[vNM] J. von Neumann and O. Morgenstern, Theory of games and economic behavior, 2d ed., Princeton University Press, 1947.

INSTITUTE FOR ADVANCEd StUdy

2 Similar results (unpublished) have been obtained by Gale, Kuhn, and Tucker independently of those of the author. An abstract, apparently motivated by consideration of matrices $B$ which have large constant segments, of these results is D. Gale, H. W. Kuhn, and A. W. Tucker, Bull. Amer. Math. Soc. Abstract 55-11-472. 